\title{
Directionally controlled superconductivity in ferromagnet/superconductor/ferromagnet trilayers with biaxial easy axes
}

\author{
C. Visani, ${ }^{1}$ N. M. Nemes, ${ }^{1}$ M. Rocci, ${ }^{1}$ Z. Sefrioui, ${ }^{1}$ C. Leon, ${ }^{1}$ S. G. E. te Velthuis, ${ }^{2}$ A. Hoffmann, ${ }^{2}$ M. R. Fitzsimmons, ${ }^{3}$ \\ F. Simon, ${ }^{4}$ T. Feher, ${ }^{4}$ M. Garcia-Hernandez, ${ }^{5}$ and J. Santamaria ${ }^{1}$ \\ ${ }^{1}$ GFMC, Depto. Fisica Aplicada III, Universidad Complutense de Madrid, Campus Moncloa, Madrid 28040, Spain \\ ${ }^{2}$ Materials Science Division, Argonne National Laboratory, Argonne, Illinois 60439, USA \\ ${ }^{3}$ Los Alamos National Laboratory, Los Alamos, New Mexico 87545, USA \\ ${ }^{4}$ Department of Physics, Budapest University of Technology and Economics, and Condensed Matter Physics Research Group, HAS, \\ P.O. Box 91, H-1521, Budapest, Hungary \\ ${ }^{5}$ Instituto de Ciencia de Materiales de Madrid, Consejo Superior de Investigaciones Cientificas, Cantoblanco 28049, Spain
}

(Received 8 February 2010; published 11 March 2010)

\begin{abstract}
We report on the magnetic anisotropy controlled modulation of the superconductivity in $\mathrm{La}_{0.7} \mathrm{Ca}_{0.3} \mathrm{MnO}_{3} / \mathrm{YBa}_{2} \mathrm{Cu}_{3} \mathrm{O}_{7-\delta} / \mathrm{La}_{0.7} \mathrm{Ca}_{0.3} \mathrm{MnO}_{3}$ ferromagnet/superconductor/ferromagnet hybrids with biaxial easy axes. The magnetoresistance (MR) is determined by the local misalignment of the magnetizations in the two layers and exhibits a positive MR plateau for antiparallel alignment along the easy axes and negative MR peaks at the coercive field near the hard axes. This evidences the importance of spin-dependent interfacial scattering effects (as opposed to stray fields) in the MR behavior of superconducting oxide inverse spin switches.
\end{abstract}

DOI: 10.1103/PhysRevB.81.094512

PACS number(s): 74.78.Fk, 73.43.Qt, 74.45.+c, 85.25.-j

\section{INTRODUCTION}

Giant magnetoresistance (GMR) in ferromagnet (F)/ normal metal $(\mathrm{N}) /$ ferromagnet structures results from the modulation of the resistance by the relative orientation of the magnetization of the F layers. ${ }^{1,2}$ When the spacer metal is a superconductor $(\mathrm{S})$, interesting new effects are expected from the interplay between both long-range orders. ${ }^{3,4}$ At F/S interfaces the superconducting order parameter penetrates short distances into the ferromagnet over which Cooper pairs are directly exposed to the exchange interaction, what suppresses superconductivity. F/S/F double junctions have attracted particular attention due to the possibility of amplified superconductivity control by the relative magnetic alignment of the F layers (parallel, P, vs antiparallel, AP), effectively modulating the suppression of $T_{c}$. The AP state was proposed to average out the effect of the exchange field over the coherence volume thus giving rise to shift of the resistance curve to higher temperatures. This results in large MR ratios $\left[\left(R_{\mathrm{P}}-R_{\mathrm{AP}}\right) / R_{\mathrm{P}}\right.$ with $\left.R_{\mathrm{P}}>R_{\mathrm{AP}}\right]$ (Refs. 5-10) extending over a narrow temperature range along the resistive transition, as opposed to GMR in F/N/F structures which is known to persist over wide temperature ranges.

Several recent reports proposed a different mechanism ${ }^{11}$ in which the $T_{c}$ is further reduced in the $\mathrm{AP}$ alignment $\left(R_{\mathrm{P}}\right.$ $<R_{\mathrm{AP}}$ ) due to quasiparticle confinement in the $\mathrm{S}$ layer resulting from spin-dependent scattering at the F/S interfaces. ${ }^{12-15}$ The terms direct superconducting spin (DSS) and inverse superconducting spin (ISS) switch were introduced to describe enhanced or depressed superconductivity for AP alignment of the magnetizations of the ferromagnetic layers. ${ }^{13}$ It has been accepted that an ISS governed by AP alignment ought to exhibit a well-defined positive plateau in the MR between the two coercive fields of top and bottom layers, ${ }^{13,14,16}$ while a DSS governed by the exchange field effect would have a negative MR. ${ }^{10}$ The domain state of the ferromagnet can also play a primary role in modulating the pairing amplitude by the (dipolar) stray fields generated by domains or domain walls ${ }^{17}$ in a way which in F/S/F structures may also be determined by magnetic alignment. ${ }^{10,16,18}$ Stray fields are expected to yield ISS in the sense of exhibiting positive MR, but instead of a plateau, MR peaks appear at the coercive fields where the domain state is formed. ${ }^{10,16,18}$ Recently, Zhu et al. ${ }^{10}$ have found negative MR in a DSS between the coercive fields while positive peaks at coercivity were attributed to stray fields.

To unravel the origin of the ISS, in this study we used a new methodology exploiting the modulation of the F/S interplay by the biaxial in-plane magnetic anisotropy of the ferromagnets that controls magnetic alignment and domain states. We show that in oxidebased $\quad \mathrm{La}_{0.7} \mathrm{Ca}_{0.3} \mathrm{MnO}_{3}(\mathrm{LCMO}) / \mathrm{YBa}_{2} \mathrm{Cu}_{3} \mathrm{O}_{7-\delta}(\mathrm{YBCO}) /$ $\mathrm{La}_{0.7} \mathrm{Ca}_{0.3} \mathrm{MnO}_{3}$ (LCMO) trilayers, there is evidence for a different, not yet reported, effect that consists of a positive MR plateau for AP alignment of the magnetization of the $\mathrm{F}$ layers and negative MR peak at the coercive field. The MR closely follows the AP alignment with a high-resistance state in the $\mathrm{AP}$ configuration and a low-resistance state under $\mathrm{P}$ alignment in ISS. This is consistent with a mechanism governing the MR in oxide inverse spin switches determined by the misalignment of the two $\mathrm{F}$ layer magnetizations. ${ }^{12,19}$

\section{EXPERIMENTAL DETAILS}

We grew F/S/F trilayers with fixed 15-nm-thick top and bottom LCMO layers while the YBCO thicknesses range between 8 and $21 \mathrm{~nm}$. Within this range of YBCO thickness all the samples exhibit similar magnetic and magnetoresistive behavior. ${ }^{19}$ The samples were grown on (100)-oriented $\mathrm{SrTiO}_{3}$ (STO) substrates by sputter deposition in pure oxygen pressure. ${ }^{12,19,20}$ Polarized neutron reflectometry (PNR) measurements were performed at the polarized neutron re- 


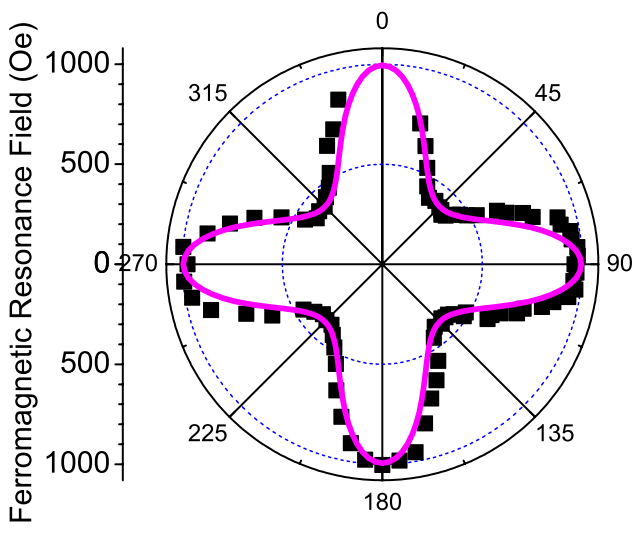

FIG. 1. (Color online) The angular dependence of the ferromagnetic resonance field (black squares) indicates a biaxial symmetry with magnetic easy axes along the [110] directions of the $\mathrm{SrTiO}_{3}$ substrate. The continuous line is a fit to Eq. (1).

flectometer ASTERIX at the Los Alamos Neutron Science Center (Los Alamos National Laboratory) to get the magnetic depth profile of the trilayers. Ferromagnetic resonance (FMR) was recorded in a commercial X-band electron spin resonance spectrometer $(9 \mathrm{GHz})$, while changing the direction of the (large) external field within the plane, above the superconducting onset. Magnetic characterization has been carried out with a vibrating sample magnetometer (VSM). Resistivity measurements were performed by using a fourprobe method, with an alternating dc current injected in plane. The plane of the samples was carefully aligned to better than $0.05^{\circ}$ in a $H=10 \mathrm{kOe}$ applied magnetic field by searching for the angle with minimal resistance, using a high-accuracy horizontal rotator. This method relies on the fact that the resistance contribution of the Abrikosov vortices is minimal when the field lies within the sample plane in thin YBCO films. Resistance vs field was measured by sweeping from a positive to a negative saturating field of $-10 \mathrm{kOe}$ and back. Magnetic field was applied at an angle $(\theta)$ with respect to the substrates edges ([100] crystallographic directions of the $\mathrm{SrTiO}_{3}$ ), which coincide with the magnetic hard axes, as shown below. For the in-plane rotational magnetoresistance experiments, the sample was mounted on a special sample holder that allows for rotating the film plane such that it remains approximately parallel to the applied field with an out-of-plane wobble of about $1^{\circ}$.

\section{RESULTS}

\section{A. Ferromagnetic resonance}

We studied the FMR at $9 \mathrm{GHz}$ by rotating the direction of the (large) external field within the film plane, on trilayer with 10 unit cells YBCO. Data were taken at $77 \mathrm{~K}$, which is above the superconducting transition temperature. The angular dependence of the FMR field of the bottom LCMO layer are shown in Fig. 1. The four minima of the FMR field (the radial value of the polar plot) along the [110] directions indicate the magnetic easy axes. We explain the data based on the following anisotropic magnetic free-energy density:

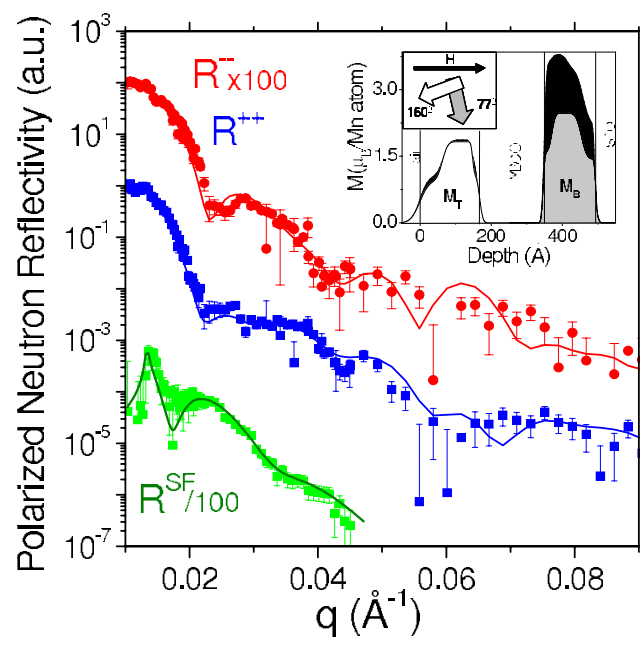

FIG. 2. (Color online) Polarized neutron reflectivity taken at 10 $\mathrm{K}$ while applying a field of 82 Oe in plane along [100]. Data (symbols) are taken with polarization analysis. A fit to the data is represented by solid lines. The inset shows the magnetic depth profile (white and gray for top and bottom layers in 82 Oe, black for both in saturation) and a schematic orientation of the magnetization in the individual LCMO layers.

$$
F=-M_{S} \mathbf{B}_{\mathbf{0}} \cdot \mathbf{m}+\left(K_{2 c}+\frac{\mu_{0}}{2} M_{S}^{2}\right) m_{z}^{2}+K_{4 a b}\left(m_{x}^{4}+m_{y}^{4}\right) .
$$

$M_{S}$ is the saturation magnetization while $\mathbf{m}$ is the unit vector of the magnetization orientation. The first term is the Zeeman energy. The second term is due to anisotropy and demagnetizing fields. They cannot be obtained separately from FMR alone, and we use $M_{S}=400 \mathrm{kA} / \mathrm{m}$ as determined by magnetometry. The last term represents the in-plane, biaxial anisotropy. The simulation in Fig. $1-$ using $K_{2 c}=+180 \mathrm{~kJ} / \mathrm{m}^{3}$ and $K_{4 a b}=+3.1 \mathrm{~kJ} / \mathrm{m}^{3}$ for magnetic anisotropies-fits the data well. Both the demagnetization and $K_{2 c}$ terms then favor an in-plane magnetization. These anisotropies may originate from single-ion anisotropies, anisotropic exchange, and, to a lesser extent, dipole-dipole interactions. This corroborates that the four [110] directions of the STO substrate are magnetic easy axes, as also found in 15-nm-thick single LCMO films grown on STO (100). ${ }^{21}$ This last issue has been discussed controversially in the literature since magnetic anisotropy, as many other properties in lanthanum manganites is strongly related to the thickness of the film, the doping and the degree of strain. ${ }^{22-30}$

\section{B. Polarized neutron reflectometry}

PNR measurements were performed to get the magnetic depth profile of the trilayers. In Fig. 2 we show the PNR (Refs. 12 and 31) of a trilayer with 18-nm-thick (15 unit cells) YBCO, taken at $10 \mathrm{~K}$ (well below the superconducting onset transition) in a magnetic field of 82 Oe. This field value, just after the first coercivity at this temperature, was applied in plane along the [100] axis (with an accuracy of $\sim 5^{\circ}$ ) after saturating in -5500 Oe. The magnetic field was 
applied along a hard axis in order to obtain a comprehensive picture of the magnetization reversal mechanism. PNR experiments were conducted with polarization analysis that allowed us to quantify the in-plane vector magnetization of the top $\left(M_{T}\right)$ and bottom $\left(M_{B}\right)$ LCMO as a function of applied field. In addition to the intensity collected for the $R^{++}$and $R^{--}$(neutron beam polarization parallel or antiparallel, respectively, to the applied field before and after reflection) spin state, a third curve $R^{\text {spin-flip }}=\left(R^{+-}+R^{-+}\right) / 2$ is shown. $R^{\text {spin-flip }}$ is the reflectivity of the portion of the neutron beam whose polarization flipped relative to the incident-beam polarization after reflection. $R^{\text {spin-flip }}$ is related to the component of the magnetization that is perpendicular to the applied field. Simultaneous analysis of the $R^{++}, R^{--}$, and $R^{\text {spin-flip }}$ curves gives detailed information not only on the magnetic depth profile but also on the angle the magnetization makes with the applied field. The fit to the data, performed using the SPIN-FLIP software, ${ }^{32}$ indicates that the angle between magnetization and applied field is $77^{\circ}$ for $M_{B}$ and $160^{\circ}$ for $M_{T}$, not the [110]-type easy axes found by FMR (see discussion of Fig. 7). The inset shows in detail the magnetic depth profile of the sample compared to the profile obtained from PNR data at a saturating field $(-5500 \mathrm{Oe})$. The magnitude of the top-layer magnetization is essentially unchanged from saturation (i.e., it rotates), whereas that of the bottom layer exhibits a significant reduction showing that there is substantial domain nucleation. Furthermore, the magnetization is reduced at the (top) surface, due to roughness, ${ }^{33}$ and at the (bottom) interface with STO due to a thin nonmagnetic ("dead") layer of about $10 \AA$. The magnetization is suppressed at both interfaces with the YBCO over a distance of 20-30 $\AA$ inside the LCMO. Concurrent structural profiles obtained by refinement of X-ray reflectivity patterns (not shown) indicates that interfacial roughness less than $10 \AA$ is not the source of this missing magnetization, and interfacial charge transfer, as discussed earlier, ${ }^{31,34}$ is responsible for this suppression of magnetization.

\section{Magnetoresistance and magnetization}

The effect of magnetic alignment on superconductivity can be closely tracked by the ensuing MR signals. Applying the magnetic field along the [110] easy axes results in a wider magnetic field interval with a better defined AP state. In Fig. 3(a) we compare an MR curve (blue circles) with the corresponding magnetic hysteresis loop (red squares), recorded with a VSM with field within the film plane, of a trilayer with 12-nm-thick (10 unit cells) YBCO. Resistance vs field and angle was measured, in a dc four-probe geometry, by saturating in $+10 \mathrm{kOe}$ and then sweeping to $-10 \mathrm{kOe}$ and back at different angles with respect to [100]. Both MR and $M(H)$ were acquired at a temperature of $T$ $=48 \mathrm{~K}$ (the zero-resistance critical temperature being $T_{c}$ $=47 \mathrm{~K})$ and at a resistance drop of $R_{\min } / R_{n} \sim 10^{-4}$. The different coercivities of bottom and top layers (100 Oe and 300 Oe, respectively) allow controlling the magnetic alignment of the F layers, which is AP to large extent in this magnetic field range. For magnetic field applied along [110] a wide positive plateau can be identified between the two distinct

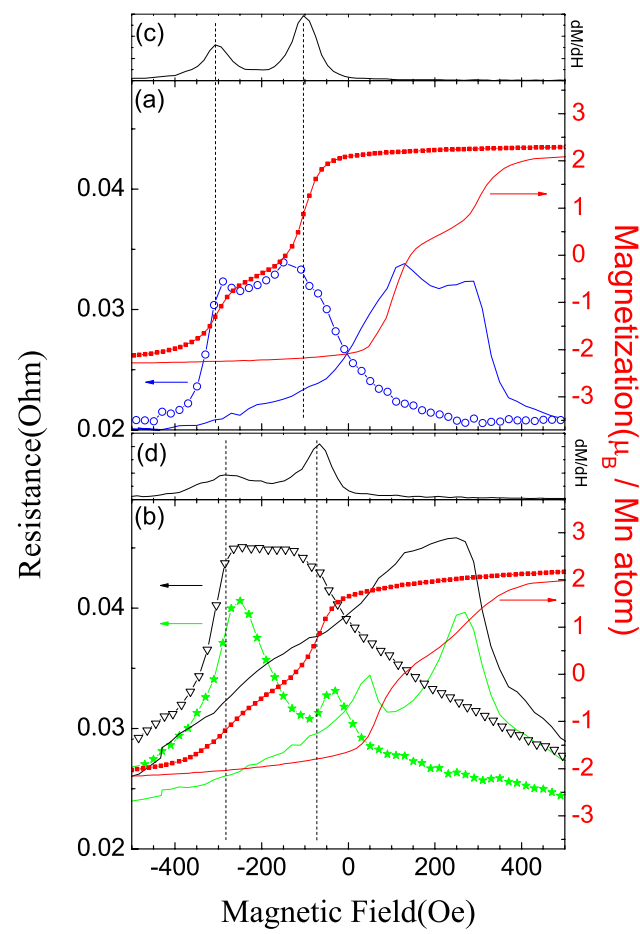

FIG. 3. (Color online) Magnetization hysteresis loops (red squares) and resistance vs field for field $H$ applied along (a) [110] (blue circles), and (b) [100] (black triangles) and $3^{\circ}$ away, in plane, from [100] (green stars). The coercive fields are indicated by dashed lines at the peak positions of the derivatives of the magnetization curves $[(\mathrm{c})$ and (d)].

coercive fields of the electrodes (marked dashed lines) where the AP alignment is maintained, clearly pointing to the importance of alignment in the MR phenomenon. When the field is applied along the [100] hard axis [see Fig. 3(b)], magnetization rotation processes become important, as indicated by MR increases well ahead of coercivity. Due to different anisotropy fields, the rotations of $M_{T}$ and $M_{B}$ are dephased, resulting in a narrow plateau located between the coercive fields with a broadened base. ${ }^{12,15,19}$ The largest MR is observed in this orientation. The shapes of the MR features do not depend on the direction of the excitation current with respect to the applied field, unlike for the AMR mechanism. ${ }^{35}$

Furthermore, small departures of $1^{\circ}-5^{\circ}$ of the applied field from the direction of the hard axes result in dips (not in peaks!) at the coercive field of the bottom layer. In the green curve in Fig. 3 and also in Fig. 6, taken at an orientation a few degrees off-hard axis, a peak appears at low field, followed first by a depression and then by the main peak. One is tempted at first sight to assign the small peak to the effect of the stray fields at coercivity. However, the coercive field coincides with the dip, not the small peak. We indicated the coercivity by the dashed line in the relevant figures throughout this paper, determined from the peaks in the derivative of the magnetization.

This slight misalignment from the hard axis is inevitably the experimental situation of the PNR experiment of Fig. 2 where significant domain nucleation was observed in the bottom layer (as the measurement was not done exactly along 


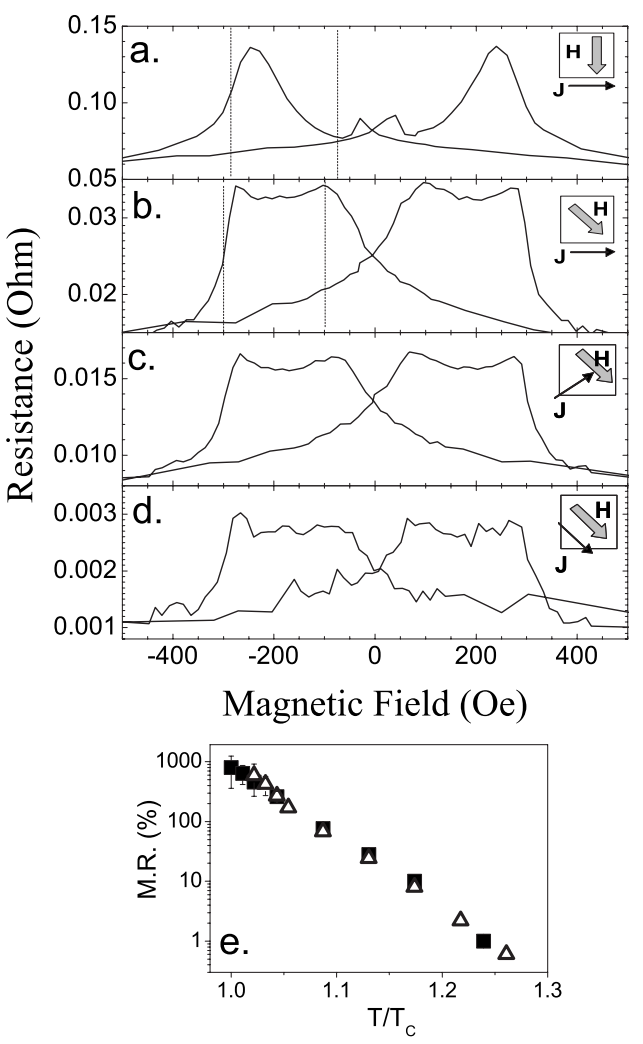

FIG. 4. Resistivity vs field for field $H$ applied along (a) $3^{\circ}$ away from [100] and (b)-(d) along [110]. Current $(J)$ direction with respect to applied field and [100] is indicated in the insets. (e) Magnetoresistance, below the superconducting onset, as a function of reduced temperature (at $T_{c}$ the resistance becomes zero), for the geometries of (a) full squares and (b) open triangles.

[100]). This new feature outlines the different behavior of our oxide system as compared to any previously reported trilayer F/S/F system based on transition metals where, typically, positive MR peaks are observed at coercivity. Negative MR peaks (dips) at coercivity in systems with perpendicular anisotropy were interpreted in terms of domain-wall-induced superconductivity, ${ }^{36-38}$ a possibility that can be ruled out in our system due to the extremely short coherence length of the cuprate. Notice also that no dips are observed for magnetic field applied along the easy axis where the largest density of domains is expected. On the other hand, stray fields generated by $\mathrm{P}$ aligned magnetostatically coupled domains are known to give rise not to negative but to positive MR peaks at coercivity.

In our case, the MR dips at the coercive field are due to $\mathrm{P}$ realignment between a fraction of domains in the bottom layer and the magnetically granular structure of the top layer (characteristic of manganites and evidenced by the reduced $M_{S}$ in Figs. 2 and 5). As we show below, positive MR is determined solely by the extent of the misalignment of the local magnetization of the two $F$ layers.

In Fig. 4, we show another set of measurements of the magnetoresistance of the 12-nm-thick YBCO trilayer sample, taken by repeatedly cooling to $48 \mathrm{~K}$. This set of data were taken in a closed-cycle refrigerator equipped with a $5 \mathrm{kOe}$ electromagnet, where the sample can be aligned manually with respect to the applied field to no better than a few degrees accuracy. In separate experimental runs, the sample was mounted with the field and excitation current applied along various directions. In panel (a) the magnetic field was oriented near, but not exactly along $[100]\left(\sim 3^{\circ}\right.$ off $)$ with the current perpendicular to field (i.e., along [010]). In panels (b)-(d) the field was applied along [110] but the contacts were remounted for each run, along [010] in (b), i.e., making $45^{\circ}$ with the applied field, along [1 $\left.1 \overline{1} 0\right]$ in (c), i.e., perpendicular to the applied field, and along [110] in (d), i.e., parallel to the applied field. The MR displays a well-defined plateau when the magnetic field is applied along [110] in panels (b)-(d), quite independent of the relative angle of the applied magnetic field and excitation current. On the contrary, when the field is applied close to [100], we observe the dips shown in green in Fig. 3 for field applied $3^{\circ}$ off [100]. These dips in most samples are well defined for the coercive field of the bottom layer only (lower $H_{c}$ ). In panel (e), we display the calculated MR values from the geometries of panels (a) as full squares and (b) as open triangles. The magnetoresistance was calculated as $\mathrm{MR}=\left(R_{\max }-R_{\text {min }}\right) / R_{\text {min }}$, where $R_{\max }$ is the maximum resistance seen between the coercive fields and $R_{\min }$ is the minimum resistance observed in the same MR sweep. The field at which the resistance becomes minimum, and thus $R_{\min }$, too, depends on the competition between the increased MR due to AP alignment that yields high resistance at low field and the vortex background that yields high resistance at high field. In Ref. 39 we have already shown that the MR in the superconducting state is not sensitive to whether the current direction is parallel or perpendicular to the applied field. Figure 4 then demonstrates that the MR is not sensitive to the in-plane direction of the applied field either, when plotted against reduced temperature. Note that the level of resistance in the four panels of Fig. 4 actually differ by almost two orders of magnitude due to the sensitivity of the superconducting transition to the exact temperature.

We compare in Fig. 5 trilayers with three different YBCO spacer thicknesses, spanning the range of samples used in the paper. Magnetoresistance (black) and magnetic hysteresis (red) data were taken with magnetic field applied along [110] in-plane axes. They show the same salient qualitative features. There is a plateau (near constant horizontal part) in the magnetization between the coercive fields of top and bottom layers and there is a corresponding large, positive magnetoresistance in this field range. There may or may not appear small peaks at the coercive fields, but the MR does not fall back to the background level. Instead the resistance stays high in-between the lower and upper $H_{c}$ 's and for this reason we refer to it as a plateau. This plateau broadens with the YBCO spacer thickness-dependent magnetic field range of AP alignment.

There are quantitative differences: the values of the coercive fields and the MR decrease monotonically with increasing YBCO spacer thickness as already shown in Ref. 19. This decrease in the value of the MR plateau was understood in terms of a model based on the diffusion of spin-polarized superconductor quasiparticles in the YBCO spacer layer. In superconductors the spin lifetime and consequently the spin- 


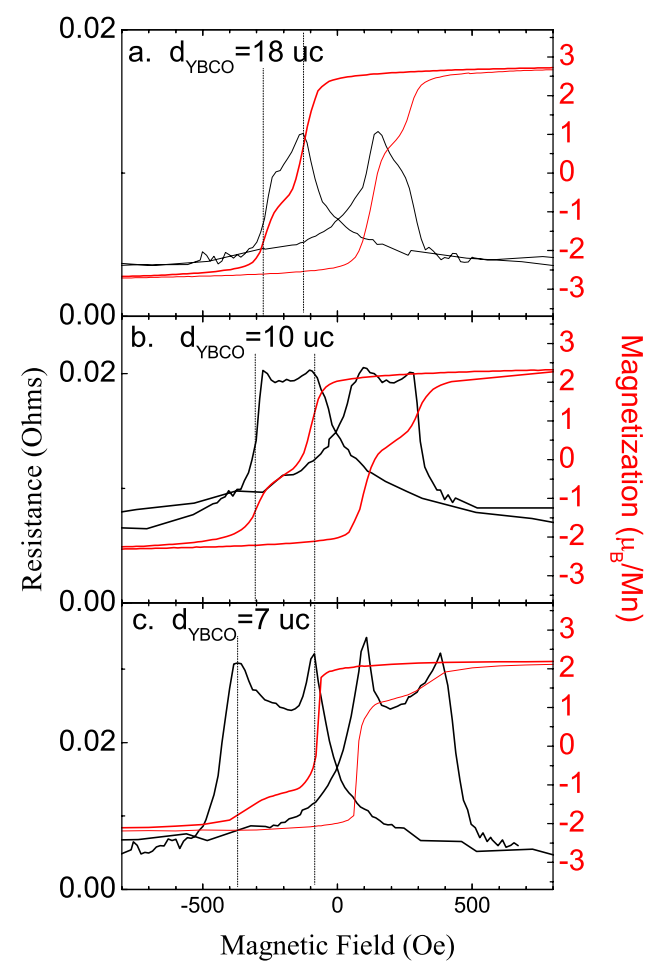

FIG. 5. (Color online) Magnetization hysteresis loops (red lines) and resistance vs field (black lines) for field $H$ applied along [110] for trilayers with YBCO thickness of (a) $21 \mathrm{~nm}$ or 18 unit cells, (b) $12 \mathrm{~nm}$ or 10 unit cells, and (c) $8 \mathrm{~nm}$ or 7 unit cells.

diffusion length increases rapidly immediately below $T_{c}{ }^{40}$ The higher coercive field corresponds to the switching of the top LCMO layer as shown by polarized neutron reflectometry (see Fig. 2 and Refs. 12 and 31). The saturation magnetization of the top layer diminishes progressively for thinner YBCO spacers as indicated by the level of the magnetization between the coercive fields. For equal magnetizations of the two layers, this level would be at $M=0$.

Figures 6 and 7 show data taken on the same trilayer with $9 \mathrm{~nm}$ YBCO spacer, at the same temperature $(T=23 \mathrm{~K})$. The sample was rotated within the cryostat in such a way that the magnetic field remained approximately within the film plane. Three MR sweeps are shown in Fig. 6, for both increasing and decreasing field: the black curve corresponds to the orientation when $H$ is along a [100] crystallographic direction or magnetic hard axis, the green curve is exactly $3^{\circ}$ away from [100], i.e., [1 $\delta 0]$ with $\delta=0.033$, while the blue curve is $45^{\circ}$ away, i.e., along [110] or magnetic easy axis. The [110] or easy axis MR shows a well-defined plateau between the coercive fields (approximately 70 and $370 \mathrm{Oe}$ ): there is no MR associated with the ferromagnetic manganite layers outside of this field region. The $[1 \delta 0] \mathrm{MR}$ does have two sharper features (peaks) near the two coercive fields. The [100] MR or hard axis data show considerably larger MR over an extended field range.

At high field (above 1000 Oe) the only MR contribution is due to the so-called vortex background that is, in turn, due to the slight out-of-plane $H$ component. It was not possible to align perfectly the rotation plane with the film plane, so there remains a minor out-of-plane field component that varies

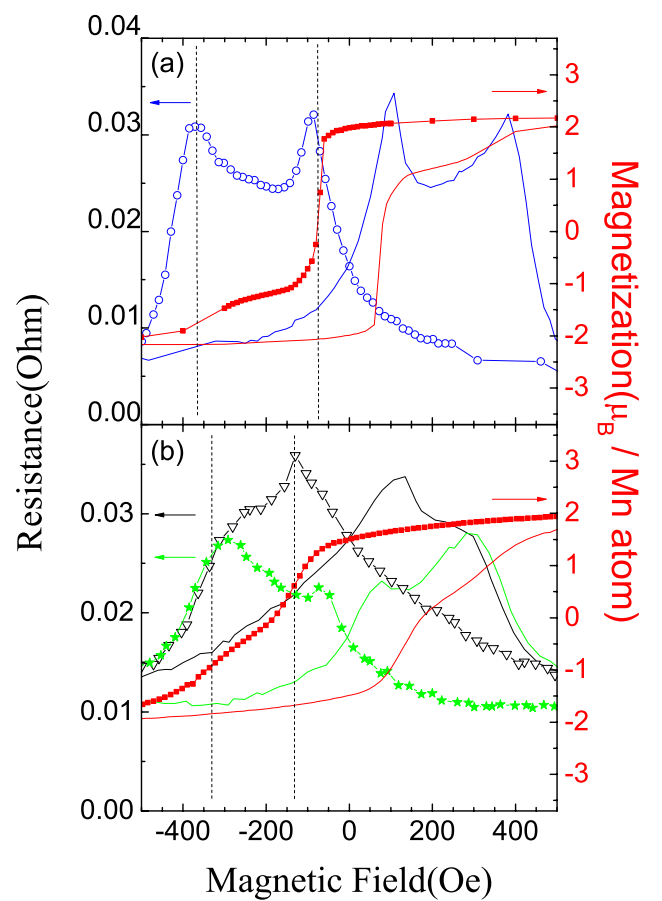

FIG. 6. (Color online) Magnetization hysteresis loops (red squares) and resistance vs field of a trilayer with 9-nm-thick YBCO for field $H$ applied along (a) [110] (blue circles) and (b) [100] (black triangles) and $3^{\circ}$ away, in plane, from [100] (green stars).

with the rotation angle. This is a small, extrinsic effect that gives rise to a varying high-field background due to the Abrikosov vortices generated in the YBCO. This misalignment issue was studied in detail by Mandal et al. in Ref. 35.

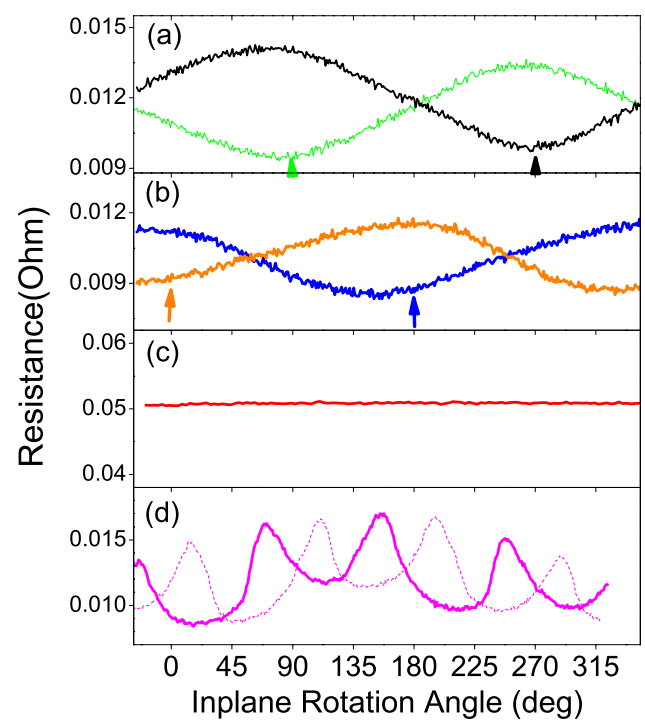

FIG. 7. (Color online) Resistance vs angle between applied field (+30 Oe) and sample edge ([100]) after saturating the sample in $-10 \mathrm{kOe}$ at an angle of (a) $90^{\circ}$ and $270^{\circ}$ (green and black lines) and (b) $0^{\circ}$ and $180^{\circ}$ (red and blue lines). Resistance vs angle in applied field of (c) +30 Oe and (d) +150 Oe after zero-field cooling. The thick lines are rotations from high angle to low angle; the thin, dashed lines show the reversal. 
Compared to the background, inferred from the [110] MR, that would be expected due only to the influence of vortices, the [100] data display considerably elevated maxima and minima. The minimum resistance is raised because of contributions to the MR due to the AP-aligned F electrodes throughout a broad field interval that extends far outside the region defined by the coercive fields. Note also, that the hard axis MR can be well approximated by a broad, single peak, although it does have a shoulder near 300 Oe (probably because even this curve was not taken exactly at [100]). Finally, the $[1 \delta 0]$ or off-hard axis MR shows a strong dip at the coercive field of the bottom LCMO at $H_{c}=120$ Oe, compared to the $[100]$ curve, rather than a peak at $\sim 60$ Oe.

\section{Magnetoresistance with in-plane magnetic field rotations}

Further information about the importance of magnetic alignment in the MR phenomenon is obtained from an experiment rotating the magnetic field in plane. The rotation experiment (using a trilayer with 9-nm-thick YBCO) was done in a small field: 30 Oe in Figs. $7(\mathrm{a})-7(\mathrm{c})$ or 150 Oe in Fig. 7(d), either after saturating at a large $-10 \mathrm{kOe}$ field, applied at an angle $\theta$ [Figs. 7(a) and 7(b)] or after zero-field cooling [Figs. 7(c) and 7(d)]. The 30 Oe field is well below the anisotropy field of the top layer, so that its magnetization $M_{T}$ remains in the direction of the saturating field (as shown by the PNR of Fig. 2) over the whole rotation sequence. On the other hand, the magnetization of the bottom layer $M_{B}$ with smaller coercivity is more sensitive to the effect of a rotation of the external field. This was shown by the PNR displaying a component of $M_{B}$ effectively pointing in a direction antiparallel to $M_{T}$ after applying a small field opposite to saturation.

Figures 7(a) and 7(b) show the results of rotations obtained after saturating at angles $\theta=0^{\circ}, 90^{\circ}, 180^{\circ}$, and $270^{\circ}$, indicated with an arrow. Notice that at the angle of initial saturation the MR is at a minimum since the top and bottom layers both point to the direction of the saturating field that establishes the $\mathrm{P}$ state. Rotation yields a cosine-like MR curve with a $360^{\circ}$ rotation-angle period. That is, for a $180^{\circ}$ field rotation, the bottom layer switches to a great extent following the field, while the top layer with a larger anisotropy field keeps pointing along the saturation direction. This indicates that the AP state of the top and bottom magnetizations is essential for the MR mechanism. The P state is established in the direction of saturation and the system keeps memory of it. The MR has a maximum at an angle $180^{\circ}$ away (opposite direction) independent of the angle of saturation. Essentially, the system keeps memory of the direction along which a large field was last applied. This unidirectional anisotropy sheds light on the puzzle of the equilibrium magnetization angles seen by PNR in Fig. 2. The magnetizations of top and bottom layers in low applied field do not settle toward the biaxial easy axes in low field, as expected, but tilt backward.

\section{DISCUSSION}

Enhanced stray fields due to magnetic flux closure through the $S$ layer have been proposed to result from mag- netostatically coupled domains in both $\mathrm{F}$ layers and have been observed to yield positive MR peaks. ${ }^{10}$ However, this mechanism requires similar coercivities of the two $F$ layers and ferromagnetically coupled face-to-face domains. This possibility can be ruled out in our system, since, as shown by magnetization and PNR, the top layer is not in a domain state at the small 30 Oe field. Moreover, 30 Oe rotation experiments in a sample cooled in zero magnetic field (when both layers end up in the domain state) show no angular modulation of the MR [see Fig. 7(c)]. This constitutes additional evidence that the angle-dependent MR is not caused by stray fields but is due to the magnetic alignment of the domain state in the top and bottom layers. In addition its $360^{\circ}$ period shows that neither is the domain state of the bottom layer at the origin of the MR, which, according to the biaxial anisotropy, would have fourfold symmetric features in the angular sweeps.

This experiment shows that although stray fields generated at domain wall may have an effect on the MR of trilayers due to superconductivity suppression, ${ }^{16,41}$ this contribution is almost completely obscured by the prevailing effect of magnetic alignment in the $\mathrm{La}_{0.7} \mathrm{Ca}_{0.3} \mathrm{MnO}_{3} / \mathrm{YBa}_{2} \mathrm{Cu}_{3} \mathrm{O}_{7-\delta} / \mathrm{La}_{0.7} \mathrm{Ca}_{0.3} \mathrm{MnO}_{3}$ trilayers. In fact, preliminary experiments on bilayer samples with rough interfaces displayed 20-40\% MR peaks; much smaller than the several hundreds (or even thousand) percent MR measured in trilayers. ${ }^{42}$ Furthermore, bilayers with improved quality yield either no MR peaks. ${ }^{19} \mathrm{MR}$ peaks originating at interface roughness has been also found in cuprate/ manganite heterostructures grown on (305)-oriented STO to promote rough growth. ${ }^{43}$

Figure $7(d)$, shows the effect of rotation of a 150 Oe field after cooling the sample in zero field. This field does modulate the domain state of the top layer (notice that the rotation is hysteretic in up and down field sweeps) and is larger than the saturation field of the bottom layer which can rotate freely. The angle-dependent MR tracks the misalignment between magnetizations when the rotating field vector crosses the hard axes. ${ }^{44}$ Notice that magnetizations of top and bottom layers are never antialigned. These results constitute solid evidence of the importance of spin-dependent effects as opposed to stray fields in yielding the MR phenomena.

In summary, we made use of the modulation of the F/S interplay by the biaxial magnetic anisotropy to examine the inverse superconducting spin-switch effect found in $\mathrm{La}_{0.7} \mathrm{Ca}_{0.3} \mathrm{MnO}_{3} / \mathrm{YBa}_{2} \mathrm{Cu}_{3} \mathrm{O}_{7-\delta} / \mathrm{La}_{0.7} \mathrm{Ca}_{0.3} \mathrm{MnO}_{3} \quad$ trilayers. When the magnetic field is applied along the easy axes we found a plateaulike giant magnetoresistance closely following the field interval over which antiparallel alignment takes place. Our insight is that, in this system, both positive and negative MR are caused by the same underlying mechanism driven by the relative alignment of the F layers, as opposed to the current belief that positive MR peaks are caused by stray fields created at magnetization switching. This result supports models based on increased spin-dependent quasiparticle scattering at the $\mathrm{F} / \mathrm{S}$ interfaces in the $\mathrm{AP}$ configuration. ${ }^{11}$ Confinement of quasiparticles due to scattering self-consistently reduces the critical temperature thus providing an explanation for the increased magnetoresistance for AP alignment of the magnetic layers. 


\section{ACKNOWLEDGMENTS}

We thank A. Goldman for fruitful discussions within the framework of the joint U.S.-Spain NSF Materials World Network under Grant No. 709584. This work was supported by the U.S. Department of Energy, Basic Energy Science, under Contracts No. DE-AC02-06CH11357 and No. DE-
AC02NA25396, by Spanish MICINN under Grant No. MAT2008-06517, Consolider Ingenio 2010 Grant CSD200900013 (Imagine), and "Ramon y Cajal" program, by CAM under Grant S2009/Mat-1756 (Phama), and by OTKA under Grants No. F61733, No. K68807, and No. PF63954, and the "Bolyai" program of the Hungarian Academy of Sciences.
${ }^{1}$ M. N. Baibich, J. M. Broto, A. Fert, F. Nguyen Van Dau, F. Petroff, P. Etienne, G. Creuzet, A. Friederich, and J. Chazelas, Phys. Rev. Lett. 61, 2472 (1988).

${ }^{2}$ G. Binasch, P. Grunberg, F. Saurenbach, and W. Zinn, Phys. Rev. B 39, 4828 (1989).

${ }^{3}$ A. I. Buzdin, Rev. Mod. Phys. 77, 935 (2005).

${ }^{4}$ F. S. Bergeret, A. F. Volkov, and K. B. Efetov, Rev. Mod. Phys. 77, 1321 (2005).

${ }^{5}$ J. Y. Gu, C. Y. You, J. S. Jiang, J. Pearson, Y. B. Bazaliy, and S. D. Bader, Phys. Rev. Lett. 89, 267001 (2002).

${ }^{6}$ A. Potenza and C. H. Marrows, Phys. Rev. B 71, 180503(R) (2005).

${ }^{7}$ I. C. Moraru, W. P. Pratt, and N. O. Birge, Phys. Rev. Lett. 96, 037004 (2006).

${ }^{8}$ F. Giazotto, F. Taddei, F. Beltram, and R. Fazio, Phys. Rev. Lett. 97, 087001 (2006).

${ }^{9}$ G.-X. Miao, A. V. Ramos, and J. S. Moodera, Phys. Rev. Lett. 101, 137001 (2008).

${ }^{10}$ J. Zhu, X. Cheng, C. Boone, and I. N. Krivorotov, Phys. Rev. Lett. 103, 027004 (2009).

${ }^{11}$ S. Takahashi, H. Imamura, and S. Maekawa, Phys. Rev. Lett. 82, 3911 (1999).

${ }^{12}$ V. Peña, Z. Sefrioui, D. Arias, C. Leon, J. Santamaria, J. L. Martinez, S. G. E. te Velthuis, and A. Hoffmann, Phys. Rev. Lett. 94, 057002 (2005).

${ }^{13}$ A. Y. Rusanov, S. Habraken, and J. Aarts, Phys. Rev. B 73, 060505(R) (2006).

${ }^{14}$ A. Singh, C. Surgers, and H. v. Lohneysen, Phys. Rev. B 75, 024513 (2007).

${ }^{15}$ K. Dybko, K. Werner-Malento, M. Sawicki, and P. Przyslupski, EPL 85, 57010 (2009).

${ }^{16}$ R. Steiner and P. Ziemann, Phys. Rev. B 74, 094504 (2006).

${ }^{17}$ Z. R. Yang, M. Lange, A. Volodin, R. Szymczak, and V. V. Moshchalkov, Nature Mater. 3, 793 (2004).

${ }^{18}$ D. Stamopoulos, E. Manios, and M. Pissas, Phys. Rev. B 75, 014501 (2007).

${ }^{19}$ N. M. Nemes, M. Garcia-Hernandez, S. G. E. te Velthuis, A. Hoffmann, C. Visani, J. Garcia-Barriocanal, V. Pena, D. Arias, Z. Sefrioui, C. Leon, and J. Santamaría, Phys. Rev. B 78, 094515 (2008).

${ }^{20}$ Z. Sefrioui, D. Arias, V. Pena, J. E. Villegas, M. Varela, P. Prieto, C. Leon, J. L. Martinez, and J. Santamaria, Phys. Rev. B 67, 214511 (2003).

${ }^{21}$ N. M. Nemes, M. Garcia-Hernandez, Z. Szatmari, T. Feher, F. Simon, C. Miller, J. Garcia-Barriocanal, F. Bruno, C. Visani, V. Pena, Z. Sefrioui, C. Leon, and J. Santamaria, IEEE Trans. Magn. 44, 2926 (2008).

${ }^{22}$ Y. Suzuki, H. Y. Hwang, S. W. Cheong, and R. B. vanDover, Appl. Phys. Lett. 71, 140 (1997).
${ }^{23}$ J. O’Donnell, M. S. Rzchowski, J. N. Eckstein, and I. Bozovic, Appl. Phys. Lett. 72, 1775 (1998).

${ }^{24}$ K. Steenbeck and R. Hiergeist, Appl. Phys. Lett. 75, 1778 (1999).

${ }^{25}$ T. K. Nath, R. A. Rao, D. Lavric, C. B. Eom, L. Wu, and F. Tsui, Appl. Phys. Lett. 74, 1615 (1999).

${ }^{26}$ M. Ziese, Phys. Rev. B 62, 1044 (2000).

${ }^{27}$ M. Mathews, F. M. Postma, J. C. Lodder, R. Jansen, G. Rijnders, and D. H. A. Blank, Appl. Phys. Lett. 87, 242507 (2005).

${ }^{28}$ T. Taniuchi, H. Kumigashira, M. Oshima, T. Wakita, T. Yokoya, M. Kubota, K. Ono, H. Akinaga, M. Lippmaa, M. Kawasaki, and H. Koinuma, Appl. Phys. Lett. 89, 112505 (2006).

${ }^{29}$ I. C. Infante, J. O. Ossó, F. Sanchez, and J. Fontcuberta, Appl. Phys. Lett. 92, 012508 (2008).

${ }^{30}$ Z. H. Wang, G. Cristiani, and H. U. Habermeier, Appl. Phys. Lett. 82, 3731 (2003).

${ }^{31}$ A. Hoffmann, S. G. E. te Velthuis, Z. Sefrioui, J. Santamaria, M. R. Fitzsimmons, S. Park, and M. Varela, Phys. Rev. B 72, 140407(R) (2005).

${ }^{32}$ M. R. Fitzsimmons and C. F. Majkrzak, Modern Techniques for Characterizing Magnetic Materials, edited by Y. Zhu (Springer, New York, 2005), pp. 107-155, Chap. 3.

${ }^{33}$ M. C. Cyrille, S. Kim, M. E. Gomez, J. Santamaria, K. M. Krishnan, and I. K. Schuller, Phys. Rev. B 62, 3361 (2000).

${ }^{34}$ M. Varela, A. Lupini, S. Pennycook, Z. Sefrioui, and J. Santamaria, Solid-State Electron. 47, 2245 (2003).

${ }^{35}$ S. Mandal, R. C. Budhani, J. He, and Y. Zhu, Phys. Rev. B 78, 094502 (2008).

${ }^{36}$ A. Y. Rusanov, M. Hesselberth, J. Aarts, and A. I. Buzdin, Phys. Rev. Lett. 93, 057002 (2004).

${ }^{37}$ W. Gillijns, A. Y. Aladyshkin, M. Lange, M. J. Van Bael, and V. V. Moshchalkov, Phys. Rev. Lett. 95, 227003 (2005).

${ }^{38}$ L. Y. Zhu, T. Y. Chen, and C. L. Chien, Phys. Rev. Lett. 101, 017004 (2008)

${ }^{39}$ C. Visani, V. Pena, J. Garcia-Barriocanal, D. Arias, Z. Sefrioui, C. Leon, J. Santamaria, N. M. Nemes, M. Garcia-Hernandez, J. L. Martinez, S. G. E. te Velthuis, and A. Hoffmann, Phys. Rev. B 75, 054501 (2007).

${ }^{40}$ N. M. Nemes, J. E. Fischer, G. Baumgartner, L. Forro, T. Feher, G. Oszlanyi, F. Simon, and A. Janossy, Phys. Rev. B 61, 7118 (2000).

${ }^{41}$ J. Albrecht, M. Djupmyr, S. Soltan, H. U. Habermeier, M. R. Connolly, and S. J. Bending, New J. Phys. 9, 379 (2007).

${ }^{42}$ T. Hu, H. Xiao, C. Visani, Z. Sefrioui, J. Santamaria, and C. Almasan, Physica B 403, 1167 (2008).

${ }^{43}$ M. van Zalk, M. Veldhorst, A. Brinkman, J. Aarts, and H. Hilgenkamp, Phys. Rev. B 79, 134509 (2009).

${ }^{44} \mathrm{M}$. Vogel and T. Mewes, Stoner-Wohlfarth astroid applet, August 28, 2009, http://www.bama.ua.edu/tmewes/Java/Astroid/ StonerAstroid.shtml 